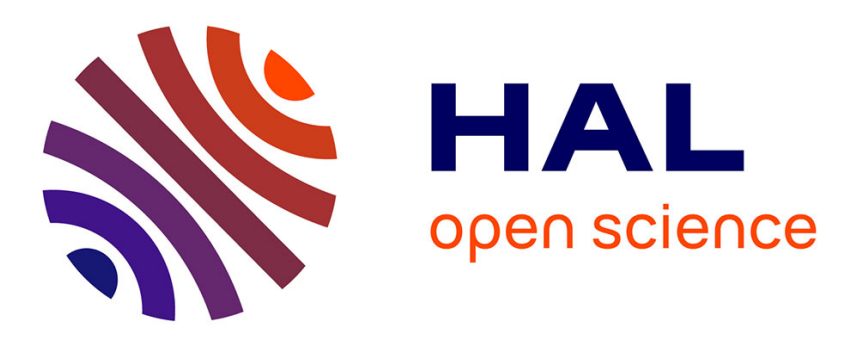

\title{
Transition Pathway between Gyroid and Cylindrical Morphology in Linear Triblock Terpolymer Thin Films
}

Aynur Guliyeva, Marylène Vayer, Fabienne Warmont, Atsushi Takano, Yushu

Matsushita, Christophe Sinturel

\section{- To cite this version:}

Aynur Guliyeva, Marylène Vayer, Fabienne Warmont, Atsushi Takano, Yushu Matsushita, et al.. Transition Pathway between Gyroid and Cylindrical Morphology in Linear Triblock Terpolymer Thin Films. Macromolecules, 2019, 52 (17), pp.6641-6648. 10.1021/acs.macromol.9b01263 . hal-03008355

\section{HAL Id: hal-03008355 \\ https://hal.science/hal-03008355}

Submitted on 16 Nov 2020

HAL is a multi-disciplinary open access archive for the deposit and dissemination of scientific research documents, whether they are published or not. The documents may come from teaching and research institutions in France or abroad, or from public or private research centers.
L'archive ouverte pluridisciplinaire HAL, est destinée au dépôt et à la diffusion de documents scientifiques de niveau recherche, publiés ou non, émanant des établissements d'enseignement et de recherche français ou étrangers, des laboratoires publics ou privés. 


\section{Transition Pathway Between Gyroid and Cylindrical Morphology in Linear}

\section{Triblock Terpolymer Thin Films}

Aynur Guliyeva ${ }^{1}$, Marylène Vayer ${ }^{1}$, Fabienne Warmont ${ }^{1}$, Atsushi Takano ${ }^{2}$, Yushu

Matsushita $^{2}$ and Christophe Sinture ${ }^{1 *}$

${ }^{1}$ Interfaces, Confinement, Matériaux et Nanostructures (ICMN) UMR 7374, CNRS-Université d'Orléans, CS

40059, F-45071 Orléans, France

${ }^{2}$ Department of Molecular \& Macromolecular Chemistry, Grad. School of Engineering, Nagoya University,

Furo-cho, Chikusa-ku, Nagoya 464-8603, Japan

KEYWORDS: Order-Order-Transition, Block Copolymer, Gyroid, Cylinder

\section{Author Information}

Corresponding author

E-mail Christophe.sinturel@univ-orleans.fr

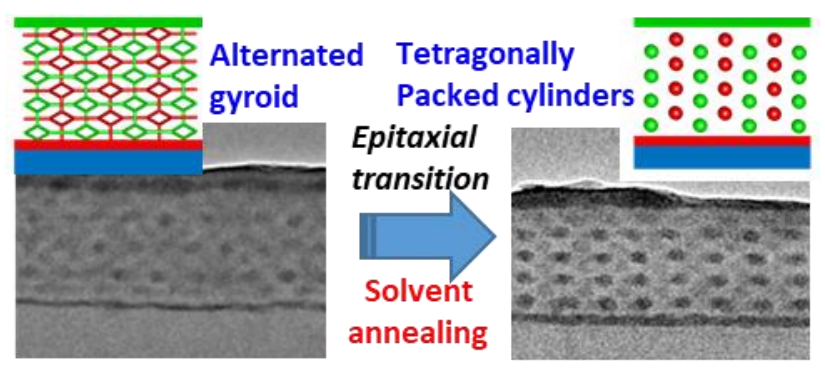




\begin{abstract}
A new transition between alternating gyroid $\left(\mathrm{G}_{\mathrm{A}}\right)$ structure and cylindrical morphology was observed in thin films for a poly(isoprene-block-styrene-block-(2-vinylpyridine)) (ISP) triblock terpolymer $(\mathrm{I} / \mathrm{S} / \mathrm{P}$ volume fraction $=0.20: 0.66: 0.14)$ exhibiting in bulk a $\mathrm{Q}_{214}$ (space group I4 ${ }_{132}$ ) morphology. Thin films were organized using solvent vapor annealing (SVA) and characterized by Atomic Force Microscopy, Transmission Electron Microscopy and Grazing-Incidence Small-Angle X-Ray scattering, coupled with several selective revealing methods adapted ISP system. After a short SVA time, the bulk morphology was reproduced with the (011) plane parallel to the surface. With a longer annealing time, tetragonal P2mm structure with cylindrical PI (polyisoprene) and P2VP (poly(2-vinylpyridine)) domains was obtained. Evidence of domains in transition between a gyroid and tetragonal structure was found, suggesting epitaxial relationship between these two structures. In contrast to the wellknown double gyroid $\left(\mathrm{G}_{\mathrm{D}}\right)$ to hexagonal (HEX) transition where $\{121\}$ planes of the $G_{D}$ structure are epitaxially related to $\{100\}$ plane of the HEX phase and cylinders grow along the $<111>$ direction, we showed that the $\{011\}$ planes of $G_{A}$ phase become the $\{001\}$ planes of cylindrical structure $(\mathrm{P} 2 \mathrm{~mm})$ and the cylinders grow along the $\langle 010\rangle$ direction of the $\mathrm{G}_{\mathrm{A}}$, parallel to the substrate. This was directly related to the three-dimensional topology of the PI and P2VP networks.
\end{abstract}




\section{Introduction}

Among the wide range of variety of morphologies formed by linear diblock and triblock copolymers, the gyroid structures have attracted a great deal of attention due to its cocontinuous nature. Such architectures display multiple domains that continuously percolate across the specimen in three dimensions (thanks to three-fold connectors), allowing for advanced mechanical, ${ }^{1}$ optical properties ${ }^{2,3}$ and exciting applications due to their high interfacial area per volume (filtration membrane, solar cells). ${ }^{4,5,6,7}$ These structures can also be used as template for elaboration of metamaterials ${ }^{2}$.

For diblock copolymers, the gyroid structure corresponds to the $I a \overline{3} d$ space group (also denoted $\mathrm{Q}_{230}$ or double gyroid $\left.\mathrm{G}_{\mathrm{D}}\right)^{8}$ with two interpenetrating networks of domains with 3-fold connectors. In the phase diagram, the gyroid phase is located between the lamellar (LAM) phase and the hexagonally packed cylinders (HEX) phase. The rather narrow window of the gyroid in the phase diagram allows for transition towards the HEX and LAM phases which can be promoted by external stimuli such as temperature, swelling, interfacial effect, shear flow and electric field. ${ }^{9,10,11}$ The $\mathrm{G}_{\mathrm{D}}$ to HEX transition has been particularly studied. In bulk, it is now well established that upon a temperature elevation, similarly to what was firstly discovered in lyotropic liquid crystals, ${ }^{12}$ the $\{121\}$ planes of the $G_{D}$ structure are epitaxially related to $\{100\}$ planes of the HEX and that the $<111>$ direction of the $G_{D}$ coincides with the cylinder axis in the HEX phase. ${ }^{13}$ Under shear flow, the cylinders still grow along the $<111>$ direction of the $\mathrm{G}_{\mathrm{D}}$ phase but another relationship between $\mathrm{G}_{\mathrm{D}}$ and hexagonally packed cylinders has been found, namely between $\{220\}$ planes of $G_{D}$ and $\{100\}$ planes of the HEX. ${ }^{14}$ Other studies ${ }^{15}$ report epitaxial relations between $\{121\} G_{D}$ and $\{100\}$ HEX and between $\{111\} G_{D}$ and $\{110\}$ HEX; $<220>G_{D}$ corresponds to the cylinder axes. In thin films, ${ }^{16}$ studies demonstrate that upon diblock cooling or heating PS-b-PI, $\{100\}$ HEX, $\{110\}$ HEX, and $\{001\}$ HEX are 
converted to $\{121\} \mathrm{G}_{\mathrm{D}},\{220\} \mathrm{G}_{\mathrm{D}}$, and $\{111\} \mathrm{G}_{\mathrm{D}}$ and vice-versa with interphase planes $\{220\}$ for $\mathrm{G}_{\mathrm{D}}$ and $\{110\}$ for HEX. Phase transition from double gyroid to hexagonally perforated lamellae in thin film of PS-b-PDMS has been recently described. ${ }^{17}$

Concerning linear triblocks, two types of gyroid have been observed following the composition in the ternary diagram. The first corresponds to the same space group $\mathrm{Q}_{230}$ as for diblocks and presents two interpenetrating networks of core-shell cylinders with 3-fold connectors. This has been observed for asymmetric compositions such as 0.40:0.41:0.19 for PI-b-PS-b-PDMS ${ }^{18}$ where the matrix is PI and the core-shell is constituted of PDMS as core and PS as shell. ${ }^{3}$ For more symmetric compositions, alternating gyroid $\left(\mathrm{G}_{\mathrm{A}}\right)$ with $I 4{ }_{1} 32$ space group (also denoted $\mathrm{Q}_{214}$ ) is generally formed with two different interpenetrating networks of each end block in a matrix of the middle block. Such morphology has been observed for PI-b-PS-b-P2VP ${ }^{19}$, PI-bPS-b-PEO $^{20}$ and for neat PI-b-PS-b-PMMA and blends of copolymer/ associated homopolymers ${ }^{21,22}$ in bulk and PI-b-PS-b-PPC ${ }^{23}$ in thin film. However, $\mathrm{Ahn}^{24}$, demonstrated that for a blend of PI-b-PS-P2VP and PI-b-PS with an overall volume fraction of 0.12:0.79:0.09 a double gyroid $G_{D}$ could be formed, the core is constituted with PI, the shell with PS and the matrix with P2VP.

In comparison to the well-known $\mathrm{G}_{\mathrm{D}} \rightarrow \mathrm{HEX}$ transition described earlier, transition from $\mathrm{G}_{\mathrm{A}}$ to cylindrical morphology has been not reported yet, to our knowledge. Recently, Dolan ${ }^{25}$ used solvent vapor annealing (SVA), to organize a $\mathrm{G}_{\mathrm{A}}$ structure for PI-b-PS-b-PEO polymer in thin film and studied the reorganization by in situ GISAXS, highlighting the different states encountered during SVA as a function of the swelling degree. Variation in the lattice parameters and long-range order has been found, but the gyroid organization was preserved among a wide range of time and swelling degree. Morphological transition from a lamellar to a $\mathrm{G}_{\mathrm{A}}$ has been observed in a PI-b-PS-b-P2VP close to the boundary of the LAM/G $\mathrm{G}_{\mathrm{A}}$ domains of the phase 
diagram $(\mathrm{I} / \mathrm{S} / \mathrm{P}$ volume fraction $=0.26 / 0.48 / 0.26) .{ }^{26}$ The purpose of this paper is to clarify the behavior of a PI-b-PS-b-P2VP polymer having a $\mathrm{G}_{\mathrm{A}}$ structure in bulk, with a composition very close to the boundary of the cylindrical phase,${ }^{27}$ submitted to a confinement effect in a thin film and its evolution during solvent vapor annealing.

\section{Materials and methods}

A poly(isoprene-block-styrene-block-(2-vinylpyridine)) triblock terpolymer (ISP) having the molecular characteristics presented in table 1 was synthesized by anionic polymerization following a protocol described elsewhere. ${ }^{28}$ This ISP block polymer (same composition and molar weight) has been already studied in bulk by SAXS and leads to $\mathrm{G}_{\mathrm{A}}$ structure (code ISP23 in ref 29). The structure was checked by TEM on a bulk specimen (see SI-1) for this study. It should be noted that the volume fraction of the center block polystyrene $\left(\varphi_{S}=0.66\right)$ is the highest one for this ISP triblock series (equal fraction of end-block polymers) leading to tricontinous morphology $\left(\mathrm{G}_{\mathrm{A}}\right)^{27,28}$ (slightly higher PS content would lead to a tetragonal arrangement of cylinders).

\begin{tabular}{|l|l|l|l|l|l|}
\hline & & \multicolumn{3}{|c|}{ Volume fraction } & \\
\hline Sample & $\mathrm{M}_{\mathrm{n}}(\mathrm{kg} / \mathrm{mol})$ & $\varphi_{\mathrm{I}}$ & $\varphi_{\mathrm{S}}$ & $\varphi_{\mathrm{P}}$ & $\mathrm{M}_{\mathrm{w}} / \mathrm{M}_{\mathrm{n}}$ \\
\hline ISP & 91 & 0.20 & 0.66 & 0.14 & 1.02 \\
\hline
\end{tabular}

Table1. Molecular characteristics of the studied ISP triblock terpolymer.

For thin films preparation, we used Si (100) substrates (kindly provided by STMicroelectronics Co) of $10 * 10 \mathrm{~mm}^{2}$, cleaned by sonication in tetrahydrofuran (THF), ethanol and acetone for 10 minutes each. Solution of ISP was prepared by dissolving polymer in solvent (1,2dichloroethane (DCE)). Then the solution was stirred for at least 2 hours and thin films were deposited by spin-coating on Si substrates at $3000 \mathrm{rpm}$ for 30 seconds. The film thicknesses, measured by visible spectral reflectometry using F20 by Filmetrics, ranged from 80 to $120 \mathrm{~nm}$. The accuracy of the determination of the thickness by visible spectral reflectometry was verified 
on polymer films using Atomic Force Microscopy (AFM) measurement of the depth of a scratch line. After deposition, the films were exposed to tetrahydrofuran (THF) vapor in a closed glass vessel (1L) containing $3 \mathrm{~mL}$ of solvent at $25^{\circ} \mathrm{C}$. All the used solvents were purchased from Sigma Aldrich and Roth Sochiel and used as received.

AFM in the tapping mode was carried out in air with AFM Dimension Icon model (Bruker ICON) and silicon cantilevers RTESP300 from Bruker were used. All the displayed AFM images are height images processed with Gwyddion. AFM characterization was also performed after selective elimination of PI domain by ozonolysis and selective expansion of P2VP by swelling in ethanol. For this purpose, thin films were exposed to ozone using a UVO Cleaner by Jelight during 5 minutes (distance between UV lamp and sample was $5 \mathrm{~cm}$ ) and rinsed in distilled water. In order to reveal the P2VP block, films were exposed to ethanol vapors in a closed Teflon vessel $(60 \mathrm{~mL})$ containing $0.2 \mathrm{~mL}$ of solvent at $25^{\circ} \mathrm{C}$ for $1 \mathrm{~min} 30 \mathrm{~s}$.

Transmission electron microscopy (TEM) was performed with a Philips CM20 operated at an accelerating voltage of $200 \mathrm{kV}$. For that purpose, films were removed from the $\mathrm{Si} / \mathrm{SiO}_{2}$ substrate by flotation at the surface of $0.5 \mathrm{M} \mathrm{NaOH}$ solution for more than $5 \mathrm{~h}$. NaOH attacks the native silica layer and leads to the detachment of film fragments from the substrate. These fragments were washed by distilled water to remove $\mathrm{NaOH}$ traces and were transferred onto a TEM grid (for top view analysis) or on a rigid plastic substrate (for sectional view analysis). The plastic substrate was covered by another rigid plastic substrate and the resulting sandwich (with the fragment to be observed in the middle) was cut by ultramicrotome. The obtained sections were transferred onto grids. All the grids were dried at RT for 1 day and were stained. Two staining steps were performed for selectively revealing the domains, namely $\mathrm{OsO}_{4}$ staining for $\mathrm{PI}$ and $\mathrm{I}_{2}$ exposure for P2VP domains. ${ }^{30}$ 
GISAXS experiments were performed on the "Xeuss" Xenocs X-ray scattering setup on thin films stained by $I_{2}$ or treated by ozonolysis to increase the electronic density contrast. The twodimensional GISAXS patterns were recorded using a high sensitivity low noise 2D detection (Pilatus 300K hybrid pixel) placed at a distance of $2329 \mathrm{~mm}$ from the sample, perpendicular to the X-ray beam (parallel beam at $8042 \mathrm{eV}$ ) with a grazing incidence angle of $0.17^{\circ}$ close to the critical angle of the films. ${ }^{31}$ The diffraction patterns were recorded and in plane GISAXS profiles were extracted.

\section{Results and Discussion}

In order to fully address the morphology of the studied block polymer in a thin film situation, we combined GISAXS, TEM and AFM analyses. In the following paragraph, we describe the results for two SVA conditions: moderate annealing time leading to a gyroid morphology and long annealing time leading to tetragonal packing of cylinders and further discuss a possible transition pathway between the two structures.

\section{Gyroid morphology}

Figure 1 displays the TEM images (top-view and cross-sectional view) of a thin film annealed for 4 hours in THF vapor, which result in both cases from the projection of a three-dimensional (3D) periodic structure. On figure 1a, after $\mathrm{I}_{2}$ exposure (selective staining of the P2VP domains) the different gray tones can be interpreted as the superposition of three-dimensional P2VP domains having different orientations inside the film. Osmium staining leads to the same type of TEM image (SI-2), although the image was even more complex since a natural contrast is obtained for P2VP without staining (PI and P2VP are observed in the image). The successive stainings (Figure 1b) lead to a combination of the two previous images. The formation of a periodic structure having a 3D structure is confirmed by the cross-sectional views (Figure 1c, d). Assuming a gyroid morphology (similar to the bulk) was formed in the film, we found that 
a $\mathrm{G}_{\mathrm{A}}$ morphology consisting of two independent networks of PI and P2VP in matrix of PS, having a (011) plane parallel to the surface is in good agreement with the TEM images described above. We will see later this assumption is confirmed by the GISAXS patterns.

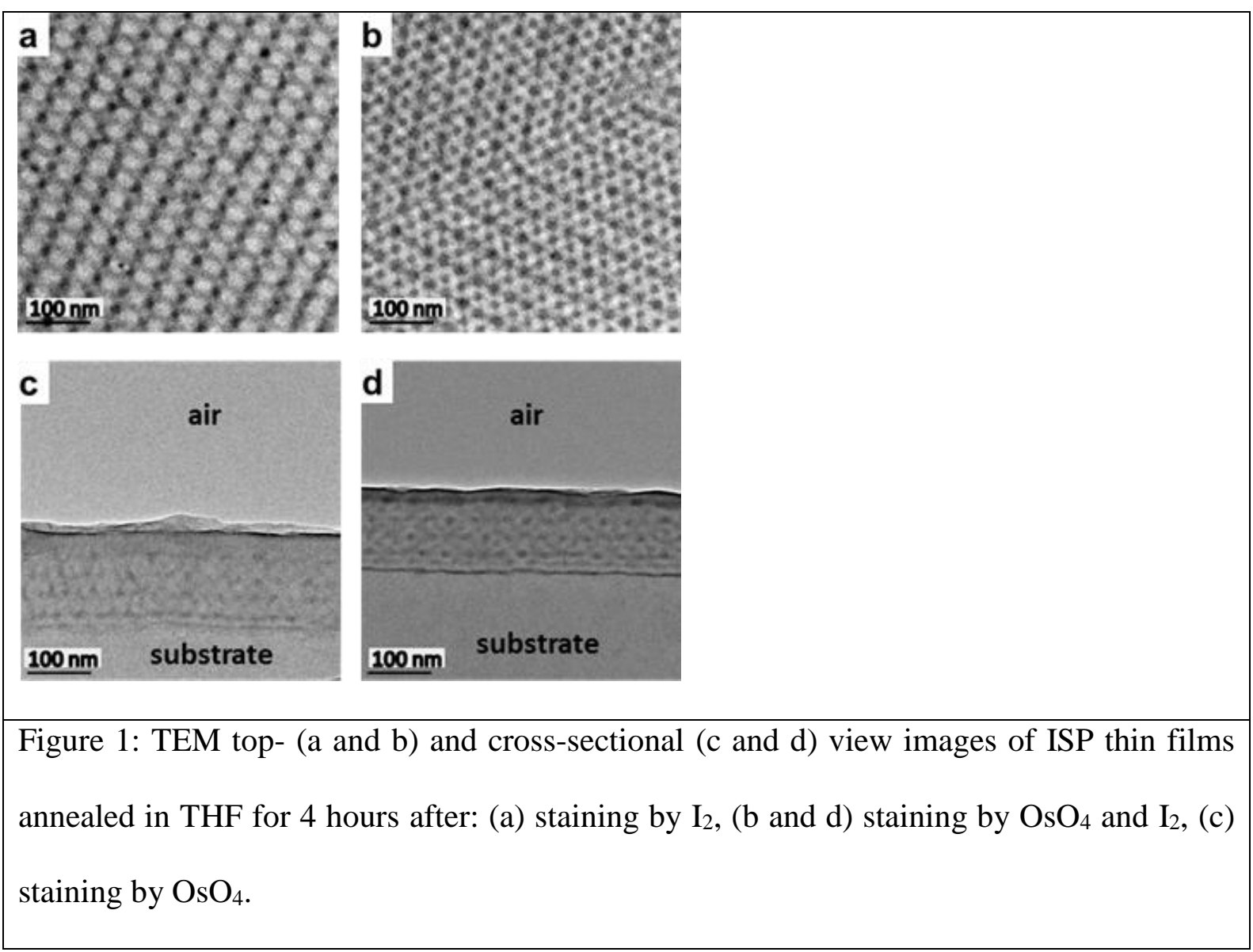


Thin film with an alternating gyroid $\mathrm{G}_{\mathrm{A}}$ structure exhibiting a (011) plane parallel to the surface was modellized using VESTA software ${ }^{32}$. Figure $2 \mathrm{a}$ and $2 \mathrm{~b}$ display the top view along the [011] direction and a particular side view (here is displayed the view along the [01-1] direction as an example). With the (011) plane parallel to the surface, one third of the three-nodes connectors of the gyroid structure are perpendicular to the surface (clearly visible on the side view of the representation, Figure $2 \mathrm{~b}$ ). Figure $2 \mathrm{c}$ displays the theoretical picture of the top-view TEM image along the [011] direction, in case of the selective staining of one domain (e.g. P2VP). Vertical stained connectors will display darker dots whereas connectors in any other directions will form lighter lines. In addition, any non-stained domains will appear white (i.e. PI and PS if P2VP is selectively stained). It can be seen that this representation matches quite well with the top-view TEM images where rows of dark gray dots connected by light gray lines were observed. On figure 2d, a successive staining of the PI and P2VP domains leads to rows of black dots (PI) alternating with rows of gray dots (P2VP).

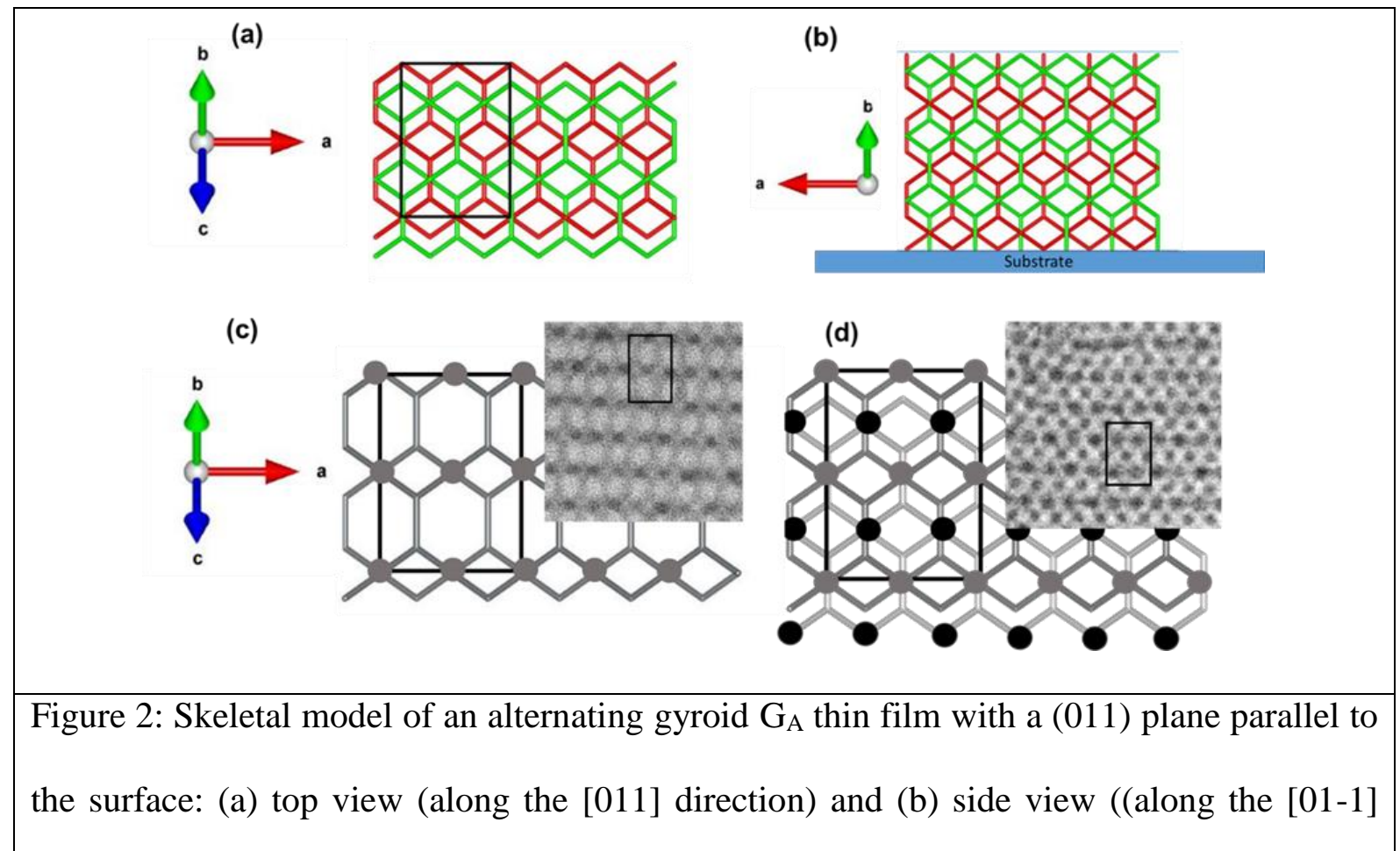


direction) (PI in green and P2VP in red)). Theoretical TEM top-view image deduced from the skeletal model: (c) considering a selective staining of the P2VP and (d) after successive staining of PI and P2VP. In inserts of (c) and (d) are shown TEM top-view images.

From the distance measured on the top-view TEM images (Figure 1a ,1b), one can extract the lattice parameter of the unit cell of the structure. As depicted in Figure 2c, the distance measured between 2 successive dots $(31 \mathrm{~nm})$ directly corresponds to the half of lattice parameter (i.e. $a / 2)$ whereas the distance measured between two rows of dots $(44 \mathrm{~nm})$ corresponds to half of the diagonal distance of the cell (i.e. $\frac{a \cdot \sqrt{2}}{2}$ ) assuming a cubic lattice where $\mathrm{a}=\mathrm{b}=\mathrm{c}$ and $\alpha=\beta=\gamma=$ $90^{\circ}$. This result was compared to the distance extracted from the cross-sectional view TEM images. As the cut could be performed in any direction perpendicular to the film surface, the corresponding images can show different types of morphologies (see SI-3 for various examples) which makes the interpretation of the images difficult. However, these images show stacks of layers containing mainly PI (or P2VP) in a matrix of PS that should be separated (according to the geometry in the cubic lattice) by a distance of $\frac{a \sqrt{2}}{2}$, i.e. $44 \mathrm{~nm}$. Instead, we measured values around $25 \mathrm{~nm}^{33}$ suggesting a contraction along the [011] direction (perpendicularly to the film substrate). Combining the characteristic average distances measured in the top/cross-sectional view images and using simple geometric consideration, we found the following parameters of the unit cell; $\mathrm{a}=62 \mathrm{~nm}, \mathrm{~b}=\mathrm{c}=50 \mathrm{~nm} \alpha=120^{\circ} \beta=\gamma=90^{\circ}$. A schematic view of the deformation of the cubic cell into the actual lattice obtained in the film is shown in the SI-4, as well as the actual top and side views of the skeletal model of the deformed $\mathrm{G}_{\mathrm{A}}$ structure under the assumed orientation (SI-5). As already observed by others ${ }^{25}$, this contraction of the lattice along the direction perpendicular to the substrate (here [011] direction) can be explained by the shrinkage 
occurring with the drying of the film during the solvent evaporation, which is directly associated with the thin film configuration. In addition to this phenomenon, the observation of the crosssectional image reveals the presence of wetting layers, which is also a specific behavior of thin films. For an osmium stained film (Figure 1c), it should be noted the absence of stained layer at the interface with the substrate and the presence of a highly stained layer at the interface with the air, indicating a wetting layer of PI at this interface. In contrast the presence of a stained layer at the interface with the substrate for osmium and iodine stained film (Figure 1d) indicates the presence of P2VP wetting layer at this interface. The presence of these wetting layers can be explained by the difference of surface energy of PI, PS, and P2VP phases $\left(\gamma_{\mathrm{I}}: 32 \mathrm{~mJ} / \mathrm{m}^{2} ; \gamma_{\mathrm{s}}\right.$ : $\left.40 \mathrm{~mJ} / \mathrm{m}^{2} ; \gamma_{\mathrm{P}}: 47 \mathrm{~mJ} / \mathrm{m}^{2}\right)^{34}$ favoring the presence of PI, compared to PS and P2VP, at the film/air interface. In contrast, P2VP is more selective than PS and PI to the substrate $\left(\gamma_{\mathrm{Si}}: 45.8 \mathrm{~mJ} / \mathrm{m}^{2}\right.$ determined using contact angles measurement of different liquids). The formation of such wetting layers at both interfaces are in good agreement with the proposed (011) orientation. Indeed, it has been shown that the planes parallel to the crystallographic (011) plane exhibit a strong modulation of the surface fraction of each component as a function of the distance from the origin, in contrast to other crystallographic planes families that exhibit a more constant composition. ${ }^{35}$ Such (011) orientation would thus lead to increased volume fraction of the minor components for given position in the volume, favoring the formation of wetting layers of PI or P2VP.

The formation of an alternating gyroid morphology $\left(\mathrm{Q}_{214}\right)$ consisting of two independent networks of PI and P2VP in matrix of PS, having a (011) plane parallel to the surface was fully confirmed by GISAXS (configuration of the GISAXS analysis is shown in Figure SI-6). GISAXS patterns were recorded after removing of PI by UVO treatment or staining of P2VP domains by $\mathrm{I}_{2}$ to increase the electronic density contrast between the blocks (Figure SI-7). 
Figure 3 shows in-plane GISAXS profiles. In both cases, the main scattering peaks appearing at $\mathrm{q}=0.14,0.24$ and $0.34 \mathrm{~nm}^{-1}$. If we consider that the film has the (011) plane parallel to the surface, in-plane peaks correspond to the scattering of the planes perpendicular to the $(011)$ film surface. The position of these most intense peaks are in good agreement with a film having a $Q_{214}$ space group and lattice parameter $a=62 \mathrm{~nm}$, the peaks being at $q / q_{0}=\sqrt{ } 2, \sqrt{ } 6, \sqrt{ } 12$.

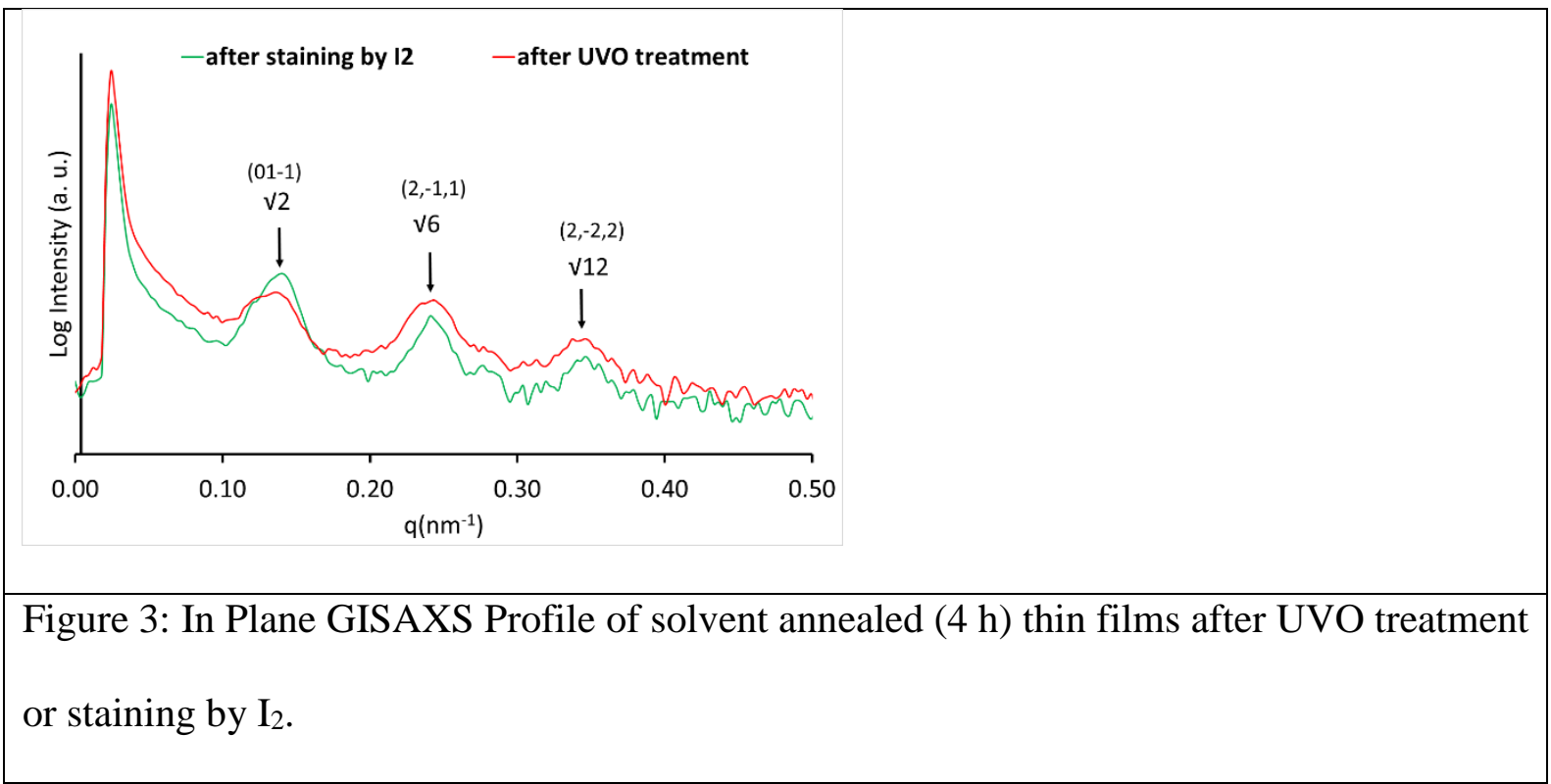

AFM was also performed on the surface of the films (Figure 4a). After solvent annealing, the AFM height image displays an apparent hexagonal packing of holes on surface, with two center-to-center distances: a short distance $(52 \mathrm{~nm})$ and a long distance $(60 \mathrm{~nm})$. After ozonolysis (UVO) (Figure 4b), a hexagonal pattern of doughnuts was observed. The exposure to ethanol vapor resulted in the selective swelling of the P2VP domains (Figure 4c) and led to a wavy type structure, the waves being distant of $52 \mathrm{~nm}$. To interpret the AFM images, it has to be kept in mind that in our case AFM was performed in tapping mode and consequently probed an interfacial layer from the surface to the near surface layer. At room temperature, PI which is above glass transition temperature, behaves like a rubber and thus is not visible during AFM scanning (a wetting layer of PI exists probably on the surface as suggested by cross- 
sectional TEM images). The interfacial layer (that we estimated $15 \mathrm{~nm}$ thick, see SI- 8 for the corresponding side view image) probed by AFM was modellized using VESTA software and the top view of the network restricted to this layer near the surface is presented in Figure 4. This view shows a pseudo-hexagonal arrangement of P2VP connectors (represented by red connectors) with in the center vertical connectors of PI (in green on the model). The hexagon center-to-center distances estimated by AFM (short $54 \mathrm{~nm}$ and long $62 \mathrm{~nm}$ ) are in good agreement with the distances calculated using VESTA software based on TEM data (long distance is $a(62 \mathrm{~nm})$ and short distance is $\left.1 / 2 * \sqrt{ }\left(\mathrm{a}^{2}+3 \mathrm{~b}^{2}\right)(53 \mathrm{~nm})\right)$. The elimination of PI on the surface by ozonolysis leads to holes in the center of the P2VP hexagons (represented by gray circles in Figure 4b) and the swelling of the P2VP leads to wavy lines (represented by wavy red thick lines in Figure 4c). This particular aspect is due to the fact that all the sides of the P2VP hexagons do not swell equally because they are not at the same surface depth (see supplementary information SI-8). All these hallmarks are in good agreement with the proposed $\mathrm{G}_{\mathrm{A}}$ structure of the polymer having its (011) plane parallel to the surface. 


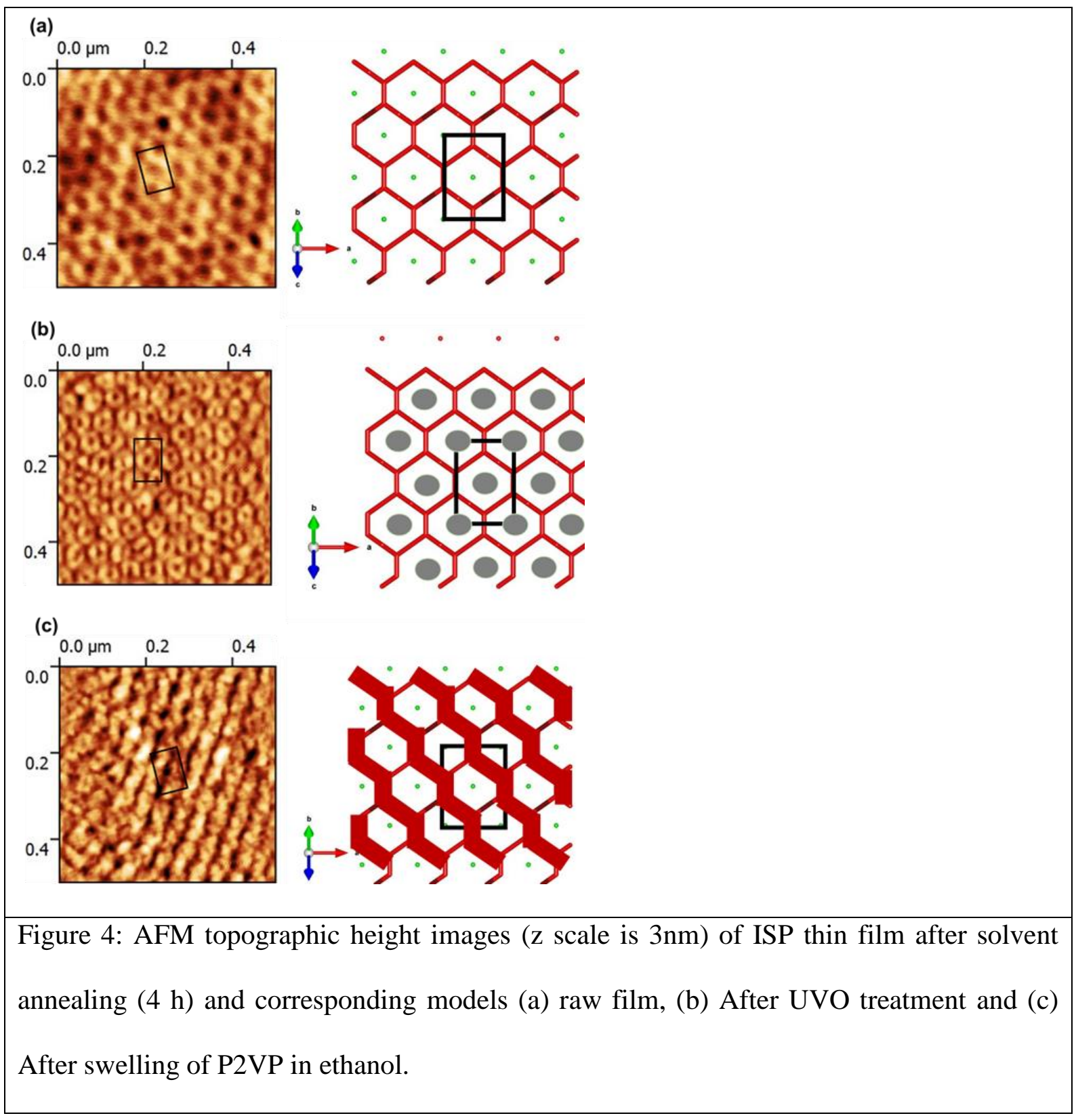

It can be concluded from this first part that, for moderate annealing time, the present ISP triblock copolymer forms a $\mathrm{G}_{\mathrm{A}}$ morphology in thin film, similarly to what was observed in the bulk. However, we found that this thin film configuration leads to some specific features that can summarized as follow:

- a preferential orientation of the $\mathrm{G}_{\mathrm{A}}$ structure, with the (011) plane parallel to the surface

- a contraction of the lattice in the [011] direction due to shrinkage that occurs during drying 
- the formation of a wetting layer of P2VP at the film/substrate interface and a wetting layer of PI at the air/film interface.

\section{Cylindrical morphology}

For longer solvent exposure time (24 h) a different kind of morphology was observed. Again, we combined AFM, TEM and GISAXS analyses to identify the new structure (Figures 5 and 6). AFM presents lines with a periodic distance of $40 \mathrm{~nm}$ (Figure 5a ,b). TEM top-view images present similar hallmarks in cases of selective stainings of PI or P2VP, consisting of dark lines distant of $41 \mathrm{~nm}$ (Figures 5c and 5d respectively). Staining PI and P2VP altogether leads to alternating black (PI), white (PS) and gray (P2VP) lines (Figure 5e). At this point it cannot be unambiguously concluded on the morphology of the system since these top-view TEM images could result from the projection of parallel cylinders or perpendicular lamellae. The morphology was fully addressed by cross-sectional view TEM images as seen in Figure 5f, revealing cylinders of PI and P2VP parallel to the substrate (SI-9 displays other cross-sectional view images). From the contrast of this image, it can be seen that all cylinders consisting of a given end component (PI or P2VP) are aligned along the vertical direction (i.e. perpendicularly to the surface), in good agreement with the top-view TEM images. The vertical distance between two cylinders of PI (or two cylinders of P2VP) was equal to ca. $20 \mathrm{~nm}$. Such arrangement corresponds to a P2mm structure whose schematic view is shown in SI-10. Again, as suggested in TEM cross-sectional view images, wetting layers of PI at air/film interface and P2VP at substrate/film interface are present (see also SI-9). 


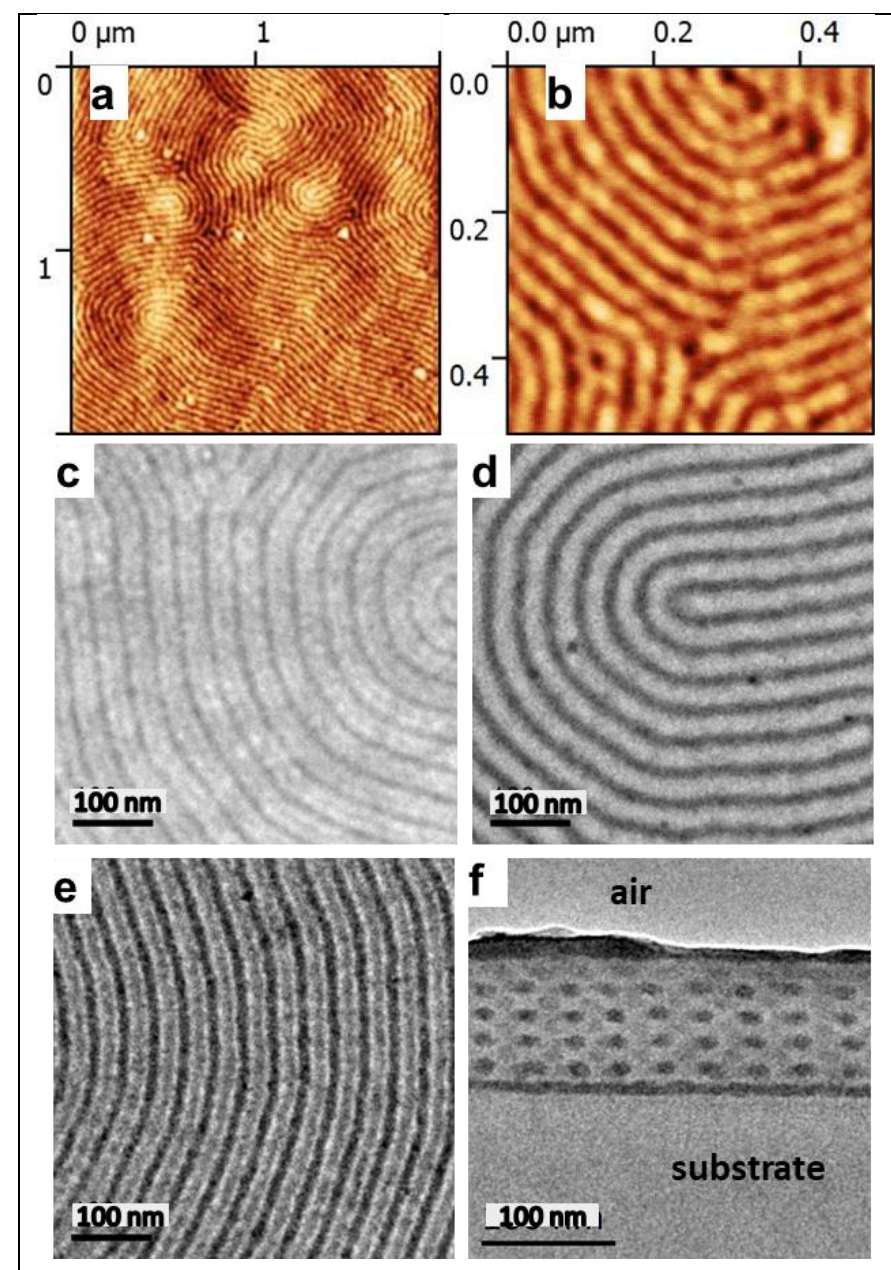

Figure 5: ISP thin films after $24 \mathrm{~h}$ solvent annealing. AFM images (a) (b). $\mathrm{Z}$ scale : $3 \mathrm{~nm}$ .TEM top-view (c, d, e) and cross-sectional view images (f) after: (c) staining $\mathrm{PI}_{\text {by } \mathrm{OsO}_{4}(\mathrm{~d})}$ staining $\mathrm{P} 2 \mathrm{VP}$ by $\mathrm{I}_{2}$, (e,f) staining by $\mathrm{OsO}_{4}$ and $\mathrm{I}_{2}$.

The morphology was confirmed by GISAXS analysis. Figure 6 show the experimental GISAXS in-plane profile extracted from the GISAXS pattern obtained for a nanostructured thin film after UVO treatment or $I_{2}$ staining (shown in SI-11). Two main scattering peaks observed for the film after $\mathrm{I}_{2}$ staining were at 0.16 and $0.32 \mathrm{~nm}^{-1}$ and correspond to scattering peaks of (100) and (200) planes perpendicular to the (001) surface. The observed value are in agreement with the observed lattice parameter $\mathrm{a}=40 \mathrm{~nm}$ of the tetragonal structure. An additional peak was 
observed after UVO treatment and corresponds to a sample where some area with $\mathrm{G}_{\mathrm{A}}$ structure remained.

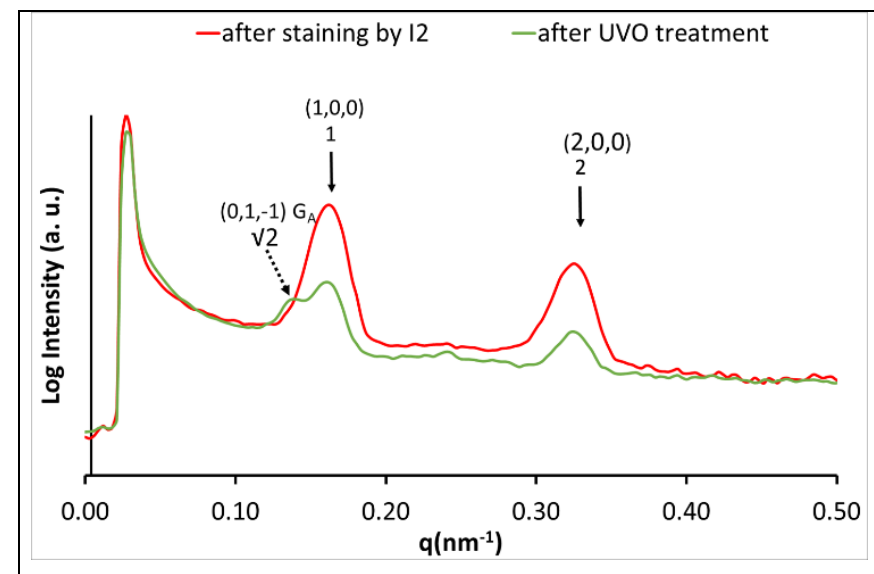

Figure 6: in-plane GISAXS profile of solvent annealed $(24 \mathrm{~h})$ thin films after staining by $\mathrm{I}_{2}$ or UVO treatment.

As mentioned earlier in the introduction and according to previous work,${ }^{27}$ the composition of this ISP block copolymer is very close to the boundary of the cylindrical phase (obtained for higher PS volume fraction). Therefore, the perturbation due the thin film configuration associated with solvent vapor annealing is sufficient to provoke a shift towards the cylindrical phase of the studied PI-b-PS-b-PVP. This is similar to what was previously observed ${ }^{26}$ with the morphological lamellar/G $\mathrm{G}_{\mathrm{A}}$ transition in a PI-b-PS-b-PVP close to the boundary of the lamellar/G $\mathrm{G}_{\mathrm{A}}$. Particularly, we think that a slightly selective swelling of the domains could be partially responsible for such behavior, ${ }^{36}$ thanks to the vicinity of the two morphologies in the phase diagram. This is in good agreement with previous measurements of the swelling of the individual components, ${ }^{37}$ showing a preferential swelling of PS compared to the P2VP, thus leading to an increased volume fraction of the central block in the swollen state. A rapid drying of the swollen film would freeze the new morphology. In addition, we postulate that the 
formation of the wetting layers, which results from the difference in surface energy of the various components, may favor the formation of a parallel array of cylinders.

\section{Transition pathway from gyroid towards cylindrical morphology}

Figure 7 presents TEM top-view images of transition zones between $\mathrm{G}_{\mathrm{A}}$ and cylinders in a P2mm structure. These zones were observed for intermediate annealing time (typically 10 hours in THF). AFM and top-view TEM images reveal the epitaxial relationship between (01-1) plane of $\mathrm{G}_{\mathrm{A}}$ and (100) plane of the cylindrical structure, [100] direction of the $\mathrm{G}_{\mathrm{A}}$ being parallel to the cylinders' axe ([010] direction) of the new structure.

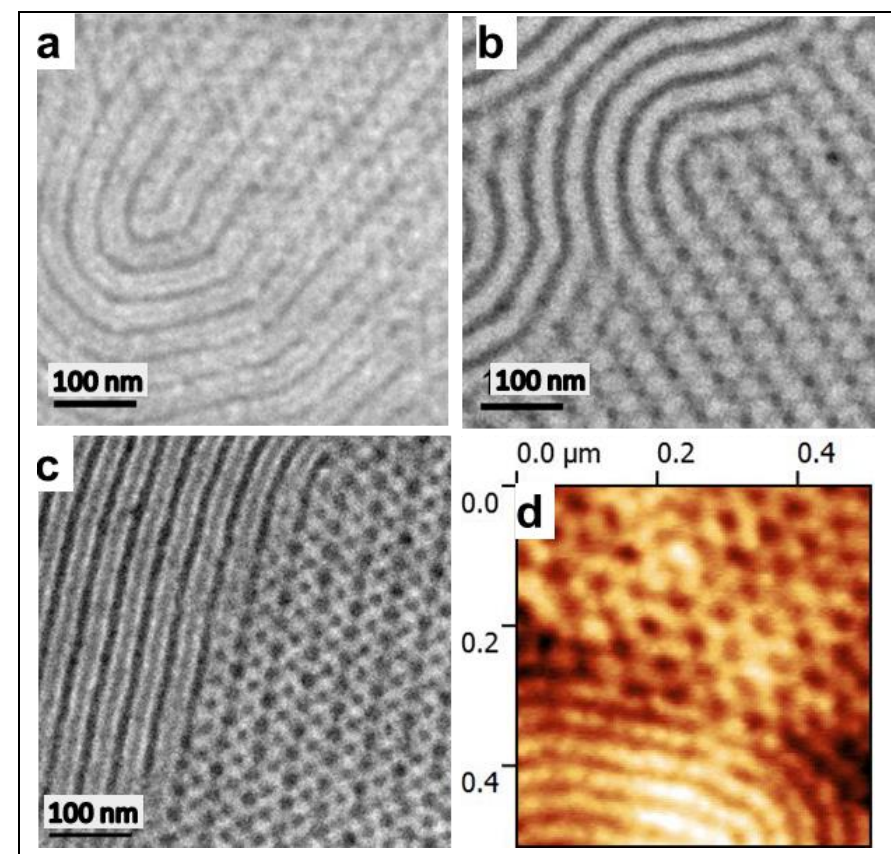

Figure 7: ISP solvent annealed (8h) thin films : TEM top-view images after (a) staining by $\mathrm{OsO}_{4}$, (b) staining by $\mathrm{I}_{2}$, (c) staining by $\mathrm{OsO}_{4}$ and $\mathrm{I}_{2}$. AFM height image (d).

The transition pathway observed here is different from the various mechanisms that has been so far proposed to describe the transition $G_{D}$ to HEX. This is because the mechanisms, which have been proposed for $G_{D}$, can simply not be applied to the $G_{A}$ structure. This can be easily 
understood if one considers for example the well-known transition pathway implying the epitaxial relationship between the $\{121\}$ planes of the $G_{D}$ and the $\{100\}$ planes of the HEX (cylinders are grown in the [111] direction of the $\left.\mathrm{G}_{\mathrm{D}}\right) \cdot{ }^{12,13}$ For one third of the newly formed cylinders, such mechanism implies the fusion of the minor domains originating from different networks $^{38}$ (see figure 8a). If this is possible for diblock polymers where the two interpenetrating networks have the same composition (red and green are equivalent in Figure 8a), this is no longer true for triblocks where the two terminal components (here, green is PI and P2VP is red) compose the two alternating networks. Such mechanism is therefore impossible in triblock terpolymer. For the same reasons, most of the other transition pathways depicted in the introduction can be discarded because they include a fusion of minor domains originating from different networks. If we now consider the [100] direction as the growth direction of the cylinders (Figure 8b), one can clearly see that each type of cylinders (PI and P2VP) will be formed separately from each network (PI and P2VP). This resembles the mode of cylinders growth described by Park et $\mathrm{al}^{15}$ in a PS- $b$-PI but in our case, the resulting arrangement of cylinders will display a P2mm symmetry, and not a typical HEX symmetry as previously observed. This specific behavior originates form the presence of three distinct components on the polymer (triblock terpolymer) that gives this unique transition path, with a completely new direction growth. This will be further confirmed by electron tomography which is a powerfull tool to perform a 3D analysis of the thin film morphology, already used by others groups on similar system. ${ }^{15,16,39}$

As a matter of characteristic distances, $b \cdot \sin (\alpha / 2)$ of the gyroid unit cell (half of the long side of the projection of the unit cell on the (011) plane) evaluated to be $44 \mathrm{~nm}$ has to be compared with the distance $a$ of the P2mm unit cell (estimated to be $40 \mathrm{~nm}$ ) and $\mathrm{b} \cdot \cos (\alpha / 2)$ of the gyroid unit cell (distance between two stacks of PI or P2VP rich layers) evaluated to be $25 \mathrm{~nm}$, has to 
be compared with the $\mathrm{c}$ distance of the $\mathrm{P} 2 \mathrm{~mm}$ unit cell (estimated to be $20 \mathrm{~nm}$ ). The comparison of these values leads to the conclusion that the transition in the swollen state between gyroid and P2mm went with a shrinkage of the unit cell of the newly formed cylindrical structure, which occurs probably during the deswelling of the film. This can be understood since in the gyroid structure, PI and P2VP domains are interlaced in 3D directions whereas in the P2mm structure, the PI and P2VP domains are oriented parallel to the surface and in one-dimensional direction. The deswelling of these two structures must not be the same, due to the enhanced mechanical properties of the $\mathrm{G}_{\mathrm{A}}$ structure.

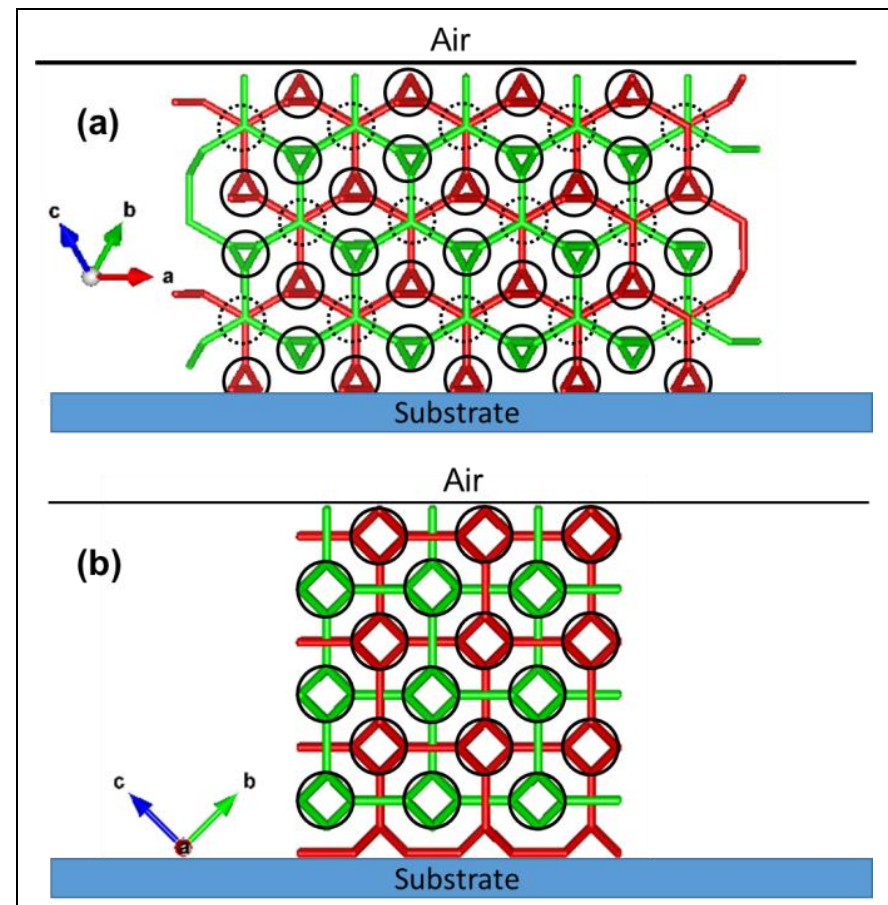

Figure 8 : The transition pathway of two different morphologies: (a) $G_{D}$ to HEX (red and green represent the same component) and (b) $\mathrm{G}_{\mathrm{A}}$ to $\mathrm{P} 2 \mathrm{~mm}$ (PI in green and P2VP in red). Black circles represent the cylinders position resulting for the reorganisation of a given type of network whereas the doted circles represent the cylinders position resulting for the fusion of two kind of networks. For clarity of the representation, the unit cell of $\mathrm{G}_{\mathrm{A}}$ and $\mathrm{P} 2 \mathrm{~mm}$ are not deformed. 


\section{Conclusion}

In conclusion, thin films of a triblock terpolymer PI-b-PS-b-P2VP, with a volume fraction of 0.20:0.66:0.14, exhibiting an alternating gyroid $\left(\mathrm{G}_{\mathrm{A}}\right)$ in bulk, could also exhibit the same structure in thin film. However, we found that this thin film configuration leads to some specific features such as a preferential orientation of the $\mathrm{G}_{\mathrm{A}}$ structure (with the (011) plane parallel to the surface), accompanied by contraction of the lattice in the [011] direction due to shrinkage which occured during drying and formation of a wetting layer of $\mathrm{P} 2 \mathrm{VP}$ at the film/substrate interface and a wetting layer of PI at the air/film interface. Long solvent annealing time led to a transition from this structure to a P2mm structure with alternating cylinders of PI and P2VP. The $\{0-11\}$ planes of gyroid become the $\{100\}$ planes of tetragonal structure and the cylinders grow along the $\langle 010\rangle$ direction parallel to the substrate. This transition has to our knowledge never been observed.

\section{Acknowledgements}

We gratefully acknowledge the financial support provided to the PIVOTS and DYSCO projects by the Région Centre - Val de Loire. Yuta Miyamori is acknowledged for his TEM contribution. Pascal Andreazza and Marie-Pierre Faugère are thanked for fruitful discussions and contribution to the GISAXS experiments.

\section{Supporting Information.}


TEM of a bulk specimen of the ISP sample staining by $\mathrm{OsO}_{4}$ and $\mathrm{I}_{2}$; Top-view TEM image of a ISP thin film annealed for 4 hours and selectively stained by $\mathrm{OsO}_{4}$; Side-view of different possible orientations of a $\mathrm{G}_{\mathrm{A}}$ morphology having the (011) plane parallel to the surface; Schematic view of the deformation of the ideal cubic lattice into the actual lattice deformed during film drying; Actual top view (011) plane (a) and side view (100) plane (b) of the skeletal model of the deformed $\mathrm{G}_{\mathrm{A}}$ structure; Configuration of the GISAXS experiment; 2D GISAXS pattern of figure 3 after (a) UVO treatment and (b) staining by $\mathrm{I}_{2}$; schematic side view image of the arrangement of the network restricted to the layer near the air surface (probed by AFM). 


\section{References}

${ }^{1}$ Potschke, P.; Paul, D. R. J. Formation of Co-continuous Structures in Melt-Mixed Immiscible Polymer Blends. J. Macromol. Sci. Polym. Rev. 2003, C43, 87-141.

${ }^{2}$ Hur, K.; Francescato, Y.; Giannini, V.; Maier, S. A.; Hennig, R. G.; Wiesner, U. Threedimensionally isotropic negative refractive index materials from block copolymer selfassembled chiral gyroid networks. Angew. Chem. Int. Edit. 2011, 50, 11985-11989.

${ }^{3}$ Dolan, J. A.; Wilts, B. D.; Vignolini, S.; Baumberg, J. J.; Steiner, U.; Wilkinson, T. D. Optical Properties of Gyroid Structured Materials: From Photonic Crystals to Metamaterials. Adv. Opt. Mater. 2015, 3, 12-32.

${ }^{4}$ Meuler, A. J.; Hillmyer, M. A.; Bates, F. S. Ordered Network Mesostructures in Block Polymer Materials. Macromolecules 2009, 42, 7221-7250.

${ }^{5}$ Okumura, A.; Nishikawa, Y.; Hashimoto, T. Nano-fabrication of double gyroid network structure via ozonolysis of matrix phase of polyisoprene in poly(2-vinylpyridine)block-polyisoprene films. Polymer 2006, 47, 7805-7812.

${ }^{6}$ Zhang, Q.; Matsuoka, F.; Suh, H. S.; Beaucage, P. A.; Xiong, S.; Smilgies, D.M.; Tan, K.W; Werner, J.G.; Nealey, P.F.; Wiesner, U. B. Pathways to Mesoporous Resin/Carbon Thin Films with Alternating Gyroid Morphology. ACS Nano 2018, 12, 347-358.

${ }^{7}$ Wang, C. Y.; Lodge, T. P. Kinetics and mechanisms for the cylinder-to-gyroid transition in a block copolymer solution. Macromolecules 2002, 35, 6997-7006. 
${ }^{8}$ Hajduk, D. A.; Harper, P. E.; Gruner, S. M.; Honeker, C. C.; Kim, G.; Thomas, E. L.; Fetters, L. J. The Gyroid: A New Equilibrium Morphology in Weakly Segregated Diblock Copolymers Macromolecules 1994, 27, 4063-4075.

${ }^{9}$ She, M.; Lo, T.; Ho, R. Controlled Ordering of Block Copolymer Gyroid Thin films Driven by Solvent Annealing. Macromolecules 2014, 47,175-182.

${ }^{10}$ Pinna, M.; Zvelindovsky, A. V. Kinetic pathways of gyroid-to-cylinder transitions in diblock copolymers under external fields: Cell dynamics simulation. Soft Matter. 2008, 4, 316.

${ }^{11}$ Jung, J.; Park, H.-W.; Lee, S.; Lee, H.; Chang, T.; Matsunaga, K.; Jinnai, H. Effect of Film Thickness on the Phase Behaviors of Diblock Copolymer Thin Film. ACS Nano 2010, 4, 31093116.

${ }^{12}$ Rançon, Y.; Charvolin, J. Fluctuations and Phase Transformations in Lyotropic Crystal Phys. Chem. 1998, 92, 6339-6344.

${ }^{13}$ Vigild, M. E.; Almdal, K.; Mortensen, K.; Hamley, I. W.; Fairclough, J. P. A.; Ryan, J. Transformations to and from the Gyroid Phase in a Diblock Copolymer. Macromolecules 1998, $31,5702-5716$.

${ }^{14}$ Honda, T.; Kawakatsu, T. Epitaxial Transition from Gyroid to Cylinder in a Diblock Copolymer Melt. Macromolecules 2006, 39, 2340-2349.

${ }^{15}$ Park, H. P.; Jung, J.; Chang, T.; Matsunaga, K.; Jinnai, H. New Epitaxial Phase Transition between DG and HEX in PS- $b$-PI. J. Am. Chem. Soc. 2009, 131, 46-47.

${ }^{16}$ Jung, J.; Lee, J.; Park, H.-W.; Chang, T.; Sugimori, H.; Jinnai, H. Epitaxial Phase Transition between Double Gyroid and Cylinder Phase in Diblock Copolymer Thin Film. Macromolecules 2014, 47, 8761-8767. 
${ }^{17}$ Wen, T.; Wang, H. F.; Georgopanos, P.; Avgeropoulos, A.; Ho, R. M. Three-dimensional visualization of phase transition in polystyrene-block-polydimethylsiloxane thin film. Polymer 2019, 167, 209-214.

${ }^{18}$ Shefelbine, T. A.; Vigild, M. E.; Matsen, M. W.; Hajduk, D. A.; Hillmyer, M. A.; Cussler E.; L.; Bates F. S. Core-Shell Gyroid Morphology in a Poly(isoprene-block-styrene-blockdimethylsiloxane) Triblock Copolymer. J. Am. Chem. Soc. 1999, 121, 8457-8465.

${ }^{19}$ Suzuki, J.; Seki, M.; Matsushita, Y. The tricontinuous double-gyroid structure from a threecomponent polymer system J. Chem. Phys. 2000, 112, 4862-4868.

${ }^{20}$ Epps III, T. H.; Cochran, E. W.; Bailey, T. S.; Waletzko, R. S.; Hardy, C. M.; Bates, F. S. Ordered Network Phases in Linear Poly(isoprene-b-styrene-b-ethylene oxide) Triblock Copolymers. Macromolecules 2004, 37, 8325-8341.

${ }^{21}$ Tureau, M. S.; Rong, L.; Hsiao, B. S.; Epps, T. H. Phase behavior of neat triblock copolymers and copolymer/homopolymer blends near network phase windows. Macromolecules 2010, 43, 9039-9048.

${ }^{22}$ Tureau, M. S.; Epps, T. H. Nanoscale networks in poly[isoprene-block-styrene-block(methyl methacrylate)] triblock copolymers. Macromol. Rapid Commun. 2009, 30, 17511755.

${ }^{23}$ Cowman, C. D.; Padgett, E.; Tan, K. W.; Hovden, R.; Gu, Y.; Andrejevic, N.; Muller, D.; Coates, G. W.; Wiesner, U. Multicomponent Nanomaterials with Complex Networked Architectures from Orthogonal Degradation and Binary Metal Backfilling in ABC Triblock Terpolymers. J. Am. Chem. Soc. 2015, 137, 6026-6033.

${ }^{24}$ Ahn, S.; Kwak, J.; Choi, C.; Seo, Y.; Kim, J. K.; Lee, B. Gyroid Structures at Highly Asymmetric Volume Fractions by Blending of ABC Triblock Terpolymer and AB Diblock 25 Copolymer. Macromolecules 2017, 50, 9008-9014. 
${ }^{25}$ Dolan, J. A.; Korzeb, K.; Dehmel, R.; Gödel, K. C.; Stefik, M.; Wiesner, U.; Wilkinson, T. D.; Baumberg, J. J.; Wilts, B. D.; Steiner, U.; Gunkel, I. Controlling Self-Assembly in Gyroid Terpolymer Films By Solvent Vapor Annealing. Small 2018, 14, 1802401.

26 Suzuki, J.; Nakane, K.; Takano, A.; Matsushita, Y. Self-assembly template during morphological transition of a linear ABC triblock copolymer from lamellar to Gyroid structure. Polymer 2004, 45, 8989-8997.

27 Matsushita, Y. Studies on Equilibrium Structures of Complex Polymers in Condensed Systems. J. Polym. Sci. B 2000, 38, 1645-1655.

${ }^{28}$ Mogi, Y.; Kotsuji, H.; Kaneko, Y.; Mori, K.; Matsushita, Y.; Noda, I. Preparation and Morphology of Triblock Copolymers of the ABC Type. Macromolecules 1992, 25, 5408-5411. ${ }^{29}$ Seki, M.; Suzuki, J.; Matsushita, Y. Small-angle X-ray scattering analysis of the periodic tricontinuous network structure of symmetric ABC triblock copolymers. J. Appl. Cryst. 2000, $33,285-290$.

${ }^{30}$ Asai, Y.; Takano, A.; Matsushita, Y. Asymmetric double tetragonal domain packing from ABC triblock terpolymer blends with chain length difference. Macromolecules 2016, 49, 69406946.

31 Cummins, C.; Mokarian-Tabari, P.; Andreazza, P.; Sinturel, C.; Morris, M. A. Solvothermal Vapor Annealing of Lamellar Poly(styrene)-block-poly(d,l-lactide) Block Copolymer Thin Films for Directed Self-Assembly Application. ACS Appl. Mater. Inter. 2016, 8, 8295-8304.

${ }^{32}$ Momma, K.; Izumi, F. VESTA 3 for three-dimensional visualization of crystal, volumetric and morphology data. J. Appl. Crystallogr. 2011, 44, 1272-1276.

${ }^{33}$ In the sectional view, the distance between the planes rich in PI could vary slightly between two samples. Since the sample is reorganized by swelling and deswelling in solvent vapors and the deswelling phenomena is quite difficult to control. 
${ }^{34}$ Guliyeva, A.; Vayer, M.; Warmont, F.; Faugère, A. M., Andreazza, P.; Takano, A.; Matsushita,Y.; Sinturel, C. Thin Films with Perpendicular Tetragonally Packed Rectangular Rods Obtained from Blends of Linear ABC Block Terpolymers. ACS Macro Lett. 2018, 7, 789794.

${ }^{35}$ Hashimoto, T.; Nishikawa, Y.; Tsutsumi, K. Identification of the "Voided Double-GyroidChannel": A New Morphology in Block Copolymers. Macromolecules 2007, 40, 1066-1072.

${ }^{36}$ Sinturel, C.; Grosso, D.; Boudot, M.; Amenitsch, H.; Hillmyer, M.A.; Pineau, A.; Vayer M. Structural transitions in asymmetric PS-b-PLA thin films induced by solvent vapor exposure. ACS Appl. Mater. Inter. 2014, 6, 12146-12152.

37 Guliyeva, A.; Vayer, M.; Warmont, F.; Faugère, A.M.; Andreazza, P.; Takano, A.; Matsushita, Y.; Sinturel C. Supporting informations of Thin Films with Perpendicular Tetragonally-Packed Rectangular Rods Obtained from Blends of Linear ABC Block Terpolymers. ACS Macro Lett., 2018, 7, 789-794.

${ }^{38}$ Matsen, M. W. Cylinder - Gyroid Epitaxial Transitions in Complex Polymeric Liquids. Phys. Rev. Lett. 1998, 80, 4470-4473.

${ }^{39}$ Mareau, V. H.; Akasaka, S.; Osaka, T.; Hasegawa, H. Direct Visualization of the Perforated Layer/Gyroid Grain Boundary in a Polystyrene-block-polyisoprene/polystyrene Blend by Electron Tomography Macromolecules 2007, 40, 9032-9039. 